\title{
Context Analysis of Health Monitoring for Patients in Care Transition Environment
}

\author{
Kiho Lee, Ronnie D. Caytiles and Sungwon Park ${ }^{*}$ \\ Hannam University, 133 Ojeong-dong, Daeduk-gu, Daejeon, Korea \\ kiholee@hnu.kr,rdcaytiles@gmail.com,*sungwon@hnu.kr
}

\begin{abstract}
The application of wireless sensor networks (WSNs) in healthcare systems has been essential to the delivery of efficient and effective healthcare services. Wireless sensor nodes frequently exchange healthcare information across different network environment as users of healthcare services are constantly on the move. The transmission of healthcare information among these sensor nodes and user devices is essentially important for an accurate and timely analysis of patient's current health condition. Thus, the need for mobility support among user devices that has a great impact on the fast delivery of healthcare information is essentially necessary. This paper deals with the context-aware analysis of an IP-enabled u-Healthcare system that utilizes wireless sensor networks (WSNs) for continuously monitoring in real-time the health condition of patients. The movement of user devices is patterned to the mobility of mobile nodes in Fast Mobile Internet Protocol version (FMIPv6) in order to handle the accurate and timely delivery of healthcare information.
\end{abstract}

Keywords: u-Healthcare system, wireless sensor networks, FMIPv6, context analysis

\section{Introduction}

Nowadays, it is essentially important to utilize wireless sensor networks (WSNs) that are capable of monitoring the current health conditions on patients. uHealthcare systems have been deploying sensor devices for measuring the different physiological information for patients with chronic diseases. The person's health condition and status are measured by these sensor devices such as glucose, breathing rate and volume, temperature, blood pressure, heart rate, and other physiological data which provides necessary information for the treatment, diagnosis, and even prevention of health problems and ailments [1]. The sensor devices can be deployed in homes, cars, workplaces, amusement parks, or healthcare centers. Other sensor devices can even be wearable or implanted on the patient's body [2]. The implementation of WSN for u-Healthcare systems addresses the drawbacks on the services that the traditional and conventional healthcare systems can offer.

The implementation of WSN in u-Healthcare systems can be enhanced through the integration of mobility support for the user mobile devices that were used in order to gather the measured physiological information by the sensor devices. The IP-enabled movements of user mobile devices can further improve the real-time monitoring of patients even remotely. With mobility support, user mobile devices can move across different network environments without impairing the delivery of healthcare information and the provision of healthcare services to the patients. The need for a seamless mobility management support for user mobile devices is essentially necessary in order to alleviate the medical treatment and diagnosis of

Received (July 15, 2017), Review Result (September 28, 2017), Accepted (November 15, 2017)

* Corresponding Author 
patients and even prevent high risk or chronic diseases such as hypertensions, diabetes, etc.

This paper aims to provide a context analysis of an IP-enabled u-Healthcare system. The IP-enabled u-Healthcare system utilizes the features of WSNs for measuring the various health conditions and status of patients to allow a remote real-time and continuous health monitoring. The advantages of Fast Mobile Internet Protocol version 6 (FMIPv6) [5] to support the mobility of user mobile devices is optimized in order to deliver a timely and accurate healthcare services. The system can predict and anticipate the movements of user mobile devices across the different network access points. The seamless connectivity and uninterrupted delivery of healthcare information is guaranteed through an efficient and optimized handover across the different wireless network environments.

The rest of this paper is organized as follows: Section 2 discusses the utilization of wireless sensor networks to u-Healthcare systems; the mobility support for user devices is outlined in Section 3; the process flow for the context-aware analysis of IP-enabled u-Healthcare system is discussed in Section 4; and the concluding remarks in Section 5.

\section{Utilization of WSNs for u-Healthcare Systems}

The utilization of Wireless Sensor Networks (WSNs) in health monitoring has been rapidly increasing. The various sensor nodes can be geographically deployed into the environment that patients are frequently located such as healthcare facilities (e.g., hospitals, healthcare centers, etc.), home, parks, or schools. Sensor nodes can even be embedded in medical instruments, equipment, or some can be wearable or embedded into patients themselves. The early detection of ailments can be provided by a continuous real-time monitoring of physiological information of patients that are delivered by these sensor nodes $[1,2]$. Thus, the effectiveness in the treatment, diagnosis, and even prevention of ailments can be achieved.

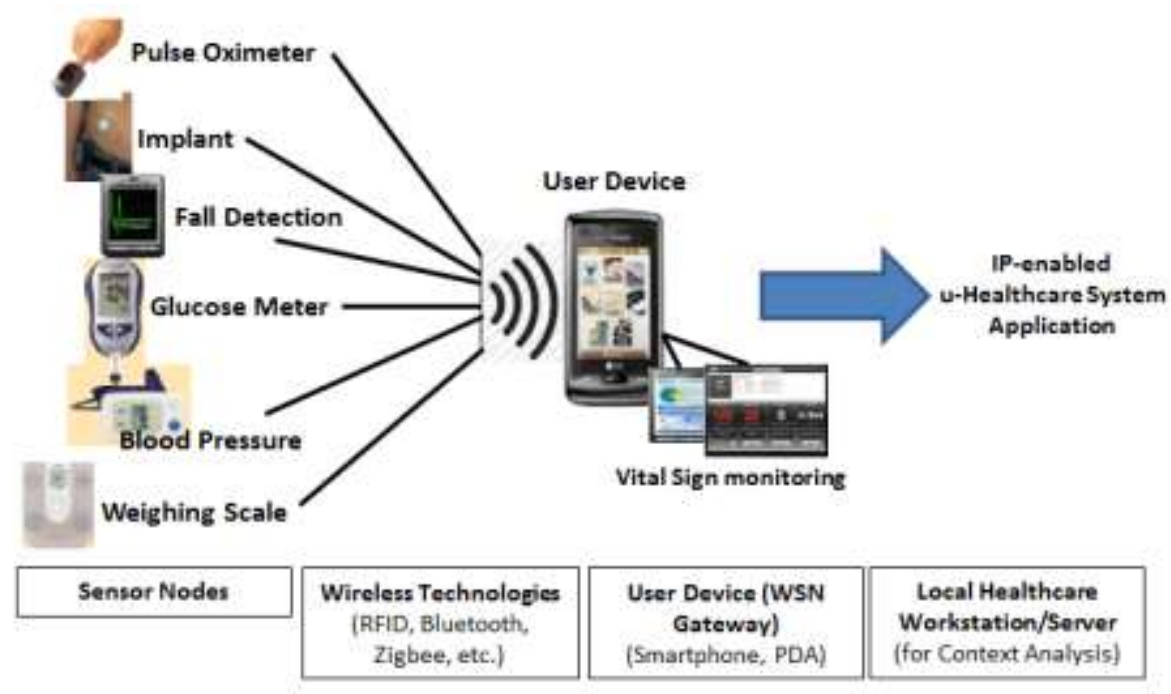

Figure 1. u-Healthcare System Employing Sensor Nodes for Real-time Monitoring

In Figure 1, the application of WSN in u-Healthcare system is shown wherein the different sensor nodes measure the essential physiological information from patients. The current health condition of patients is continuously monitored in real-time as the sensor nodes are frequently transmitting the physiological information signals to 
its base station and relayed to a monitoring device. These physiological signals are then analyzed and stored as basis for the treatment, diagnosis, and prevention of ailments.

The configuration for the utilization of WSN in healthcare system shows that the various sensor nodes transmit the measured physiological information signals into the user device that acts as its base station. The user device then receives and processes these signals in order to analyze the current health condition of patients. The physiological information is then forwarded to the healthcare server for further analysis and storage. The communication among wireless sensor nodes could utilize the advantages of RFID, Bluetooth, Zigbee, or mmWave wireless technologies.

\section{Mobility for u-Healthcare Systems}

As the patients and healthcare personnel are constantly on the move, their user device also traverses from different network environments. The patient's user device may continuously connect or disconnect from different network domains that could cause the continuity of the healthcare service to suffer. This issue can be a great factor for the efficiency of the delivery of accurate and timely information which is very essential for the real-time monitoring of health condition of patients. Thus, the need for a fast and reliable network mobility support for the management of movements of patient's user devices is essentially necessary.

Since the information that is shared across user devices is essentially important for the delivery of accurate and timely treatment, diagnosis and even prevention of ailments and chronic diseases of patients, the transfer of point of attachment (PoA) of user devices from one network to another must be seamless and with a minimal delay. These can be achieved through the application of the advantages of Fast Mobile Internet Protocol version 6 (FMIPv6) [5] on the implementation of WSN into the u-Healthcare system. The mobile node $(\mathrm{MN})$, that is, the user devices in the IP-enabled u-Healthcare system needs to partake in the mobility signaling which is related to its movement across the different network environment in order to support its mobility. It includes movement detection signaling messages, Router Solicitation (RtSol) signaling requests, Duplicate Address Detection (DAD), Binding Update (BU) signaling messages, etc.

The standard Mobile Internet Protocol version 6 (MIPv6) provides mobility support and enables a continuous communication for the mobile node (MN) and its corresponding nodes (CNs) though it is moving in a different network environment within the Internet domain [1,2]. This is done through providing the MN with two addresses: the permanent home address (HoA) in its home network; and the temporary care-of address (CoA) whenever it visits another network environment. A new CoA is issued to the MN whenever it transfers its point of attachment (PoA) and has a binding to the MN's permanent HoA. Thus, the IP packets intended to the MN will be forwarded to the MN's CoA whenever that it is away from its home network. In this regard, mobility support is given the $\mathrm{MN}$; however, the $\mathrm{MN}$ is required to inform its home agent (HA) whenever it moves into a different network environment. That is, the MN sends a binding update (BU) message to the HA that contains its new CoA where the IP packets will be forwarded to. In this case, it requires multiple exchanges of messages between the MN and HA which causes higher handover latency. 


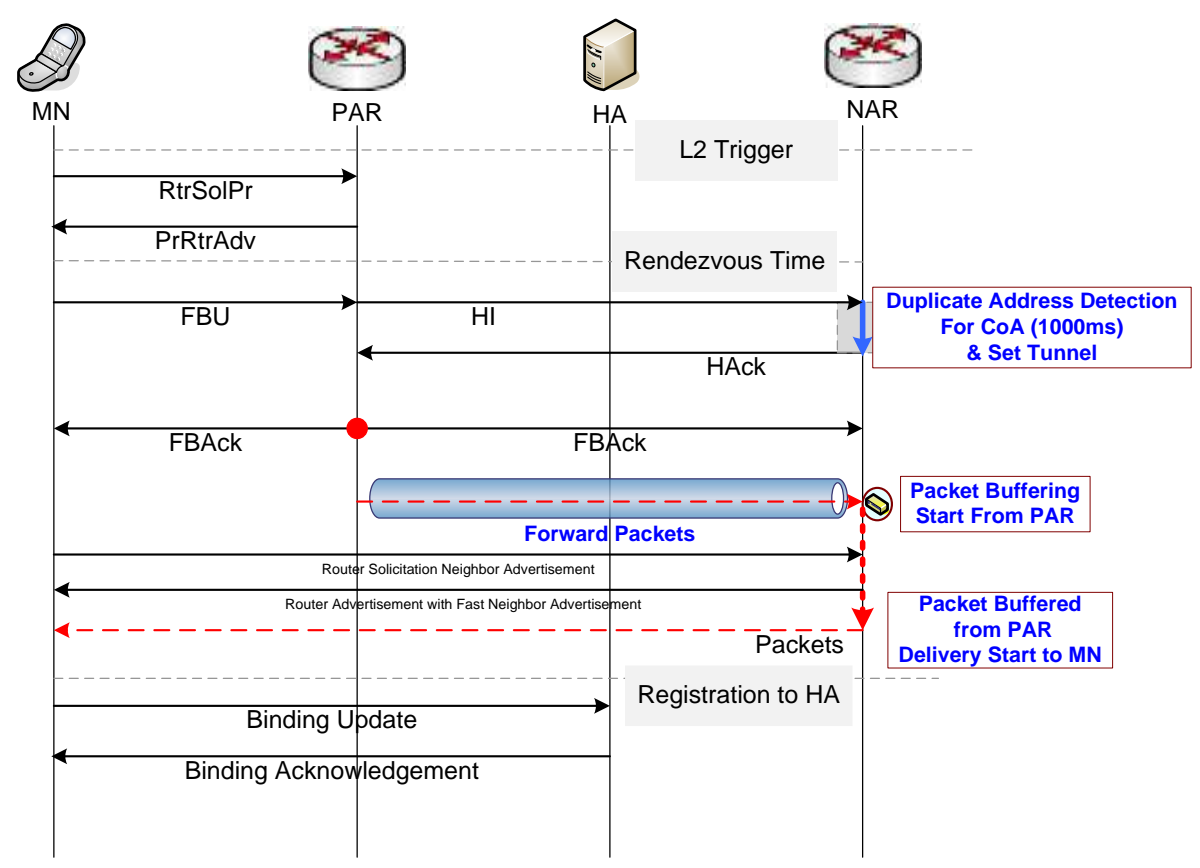

Figure 2. Fast Mobility Management for IPv6 Operations

Due to the higher latency of the standard MIPv6 caused by a number of signaling messages to be exchanged by the MN, the FMIPv6 was proposed in order to address these issues [5,6]. Through the link layer trigger events, the handover performance is improved by anticipating the movement of $\mathrm{MN}$ from one access network to another. Thus, the interruption on the delivery of IP packets will be minimized as well as the packet loss will be decreased. Figure 2(b) illustrates the functional operations for FMIPv6. The functional operations of FMIPv6 were summarized as follows: (1) Router solicitation for proxy advertisement (RtSolPr) message is sent to the previous access router (PAR) to anticipate the movement of $\mathrm{MN}$ and to request for a CoA. (2) The PAR sends back a proxy router advertisement (PrRtAdv) message with the new CoA. This address will be used by the MN whenever it is attached to the link of the new access router (NAR). The PrRtAdv message is also periodically sent by access routers. (3) The MN instigates the fast handover through the transmission of fast binding update (FBU) message which contains the acquired new CoA. This message prompts the PAR that the IP packets of the MN need to be forwarded to the NAR. (4) A tunnel needs to be established between the PAR and NAR. Thus, a handover initiate (HI) message is sent by the PAR to the NAR in order to perform duplicate address detection (DAD). (5) The NAR replies with a handover acknowledge (HAck) message and the tunnel will be successfully established whenever there is no address duplication. (6) To complete the handover process, a fast binding acknowledgement (FBack) message is sent by the PAR to the MN and NAR. The IP packets of the MN can then be forwarded to the NAR. 


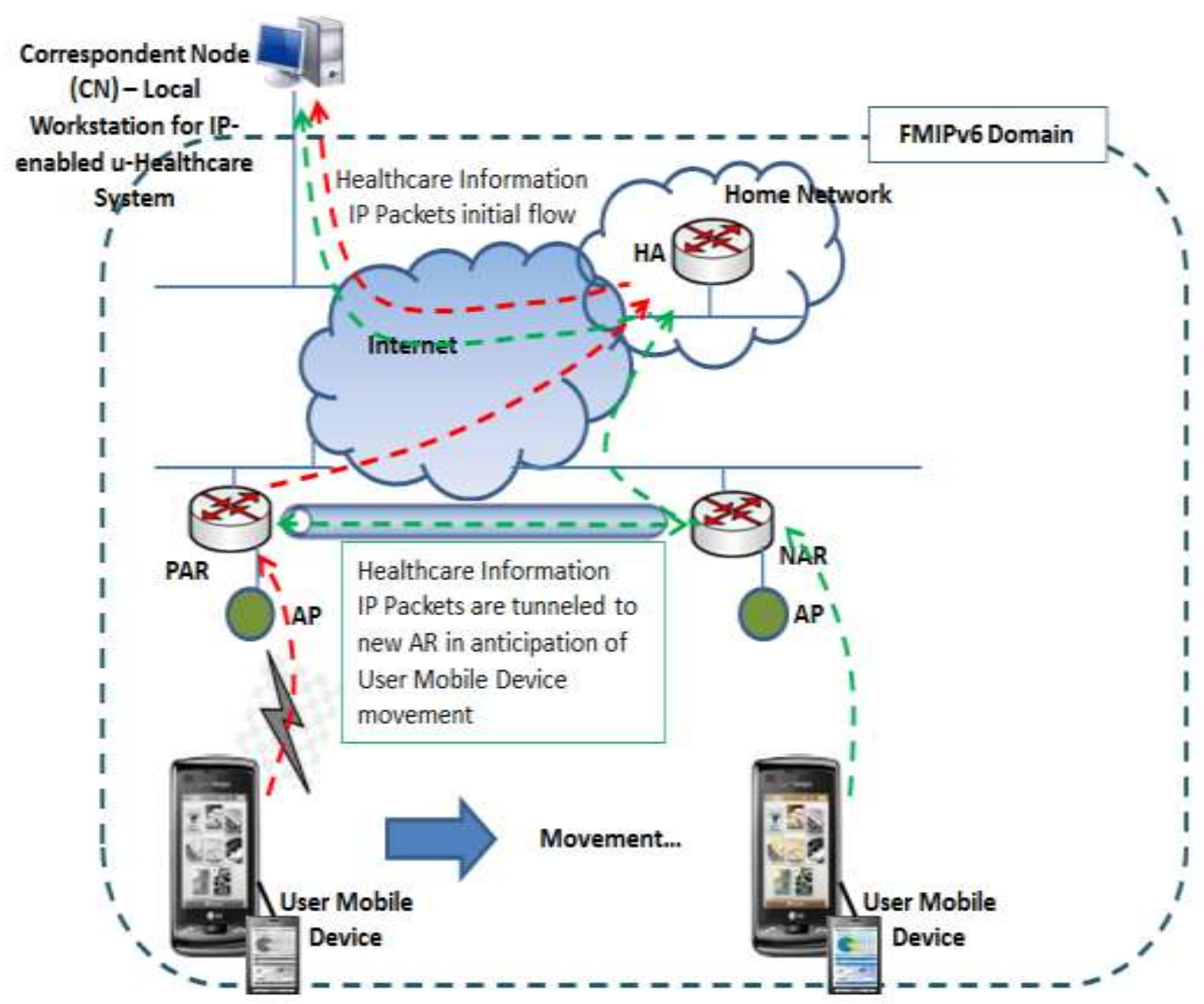

Figure 3. Mobility Support for User Devices in IP-enabled u-Healthcare System

The mobility support for the IP-enabled u-Healthcare system is patterned with the features of FMIPv6 as shown in Figure 3. That is, FMIPv6 optimizes the handover latency of user devices whenever moves across different network environments. FMIPv6 addresses the high handover latency, high packet loss, and signaling overheads for the standard MIPv6 through accurate handover prediction and anticipation of the movements of user devices. Thereby, the delivery of the essential health information for patients can be timely analyzed and the treatment, diagnosis, and even the prevention of ailments or chronic diseases will be effectively provided. The continuous and real-time monitoring of the current health condition of patients can also be provided with minimal interruption and delay.

\section{IP based u-Healthcare System Process Flow}

Health information are collected and aggregated using IP-enabled user mobile devices which is connected to the wireless sensors, and then the collected and aggregated health information are conveyed to the analyzing phase for analysis, monitoring, and storage. Healthcare personnel are alerted and notified with the current status and condition of monitored patients and necessary medical services based on patient's status and conditions are provided pervasively. This section outlines the operation of the IP-enabled u-Healthcare system which is outlined into three phases: (1) the registration phase; (2) the data gathering phase; (3) the context analysis phase. 


\subsection{Registration Phase}

The users of the IP-enabled u-Healthcare system need to register in the system. These include the patients, doctors, healthcare personnel and staff. The healthcare equipment, and medical instruments and supplies also need to be listed in the system. Each user (e.g., patients, doctors) will be provided a unique user ID that will be used as their identifier and key to his corresponding information stored in the Central Database Server. The unique user identification number for the patient will be used to associate with the sensor nodes that are attached into his body or deployed within his environment (i.e., reachable by the range of its user mobile device). The patient identification number will first be authenticated and verified in the local workstation located in healthcare centers through matching it to the Central Database Server. If the patient identification number exists, then it starts to process the corresponding data of that particular patient. The registration phase is depicted in Figure 4.

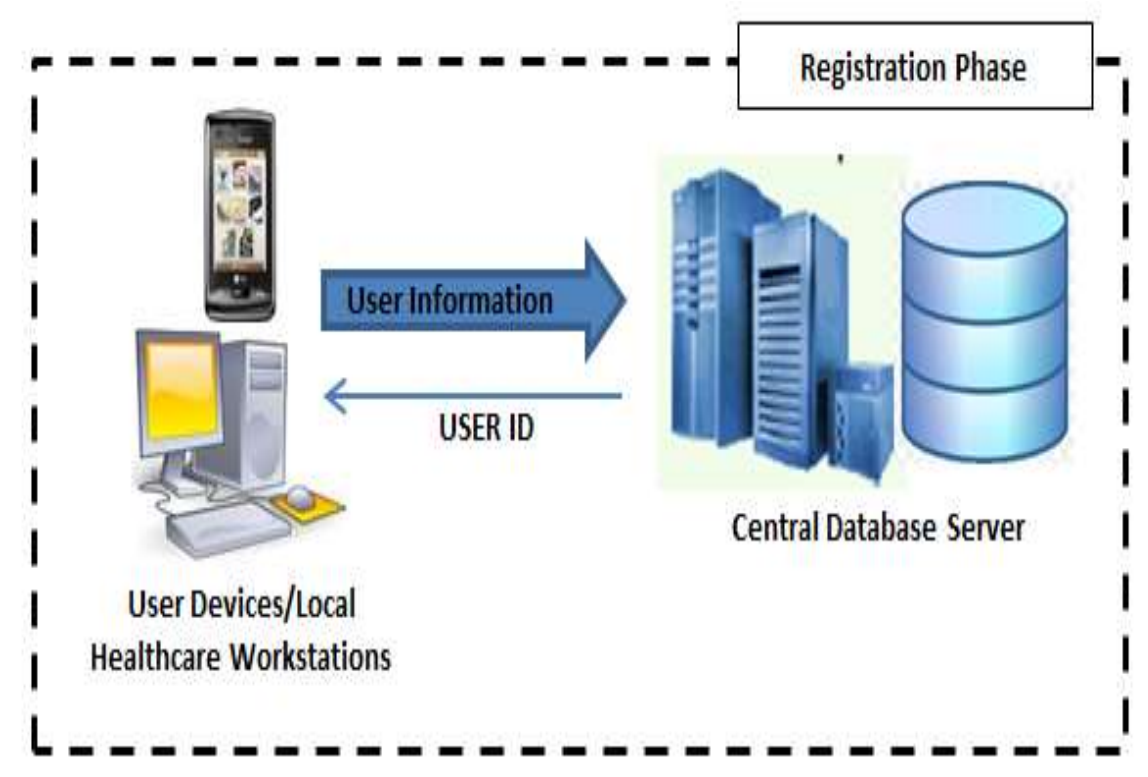

\section{Figure 4. Registration Phase}

\subsection{Data Gathering Phase}

The various sensor devices that are worn or implanted on the registered patients and those deployed within his environment measures the different physiological information in order to determine his current heath condition and status and can be regularly monitored even remotely. This physiological information gathering is performed in the data gathering phase as shown in Figure 5. The physiological information is conveyed through wireless technologies such as RFID, Bluetooth, Zigbee, or NFC to the user mobile device. This phase performs the measurement and gathering of the different physiological information, aggregates and pre-process (e.g., signals are converted into a single data format) the collected physiological information, and transmits these information to the local workstation located in the healthcare centers or vehicles. 


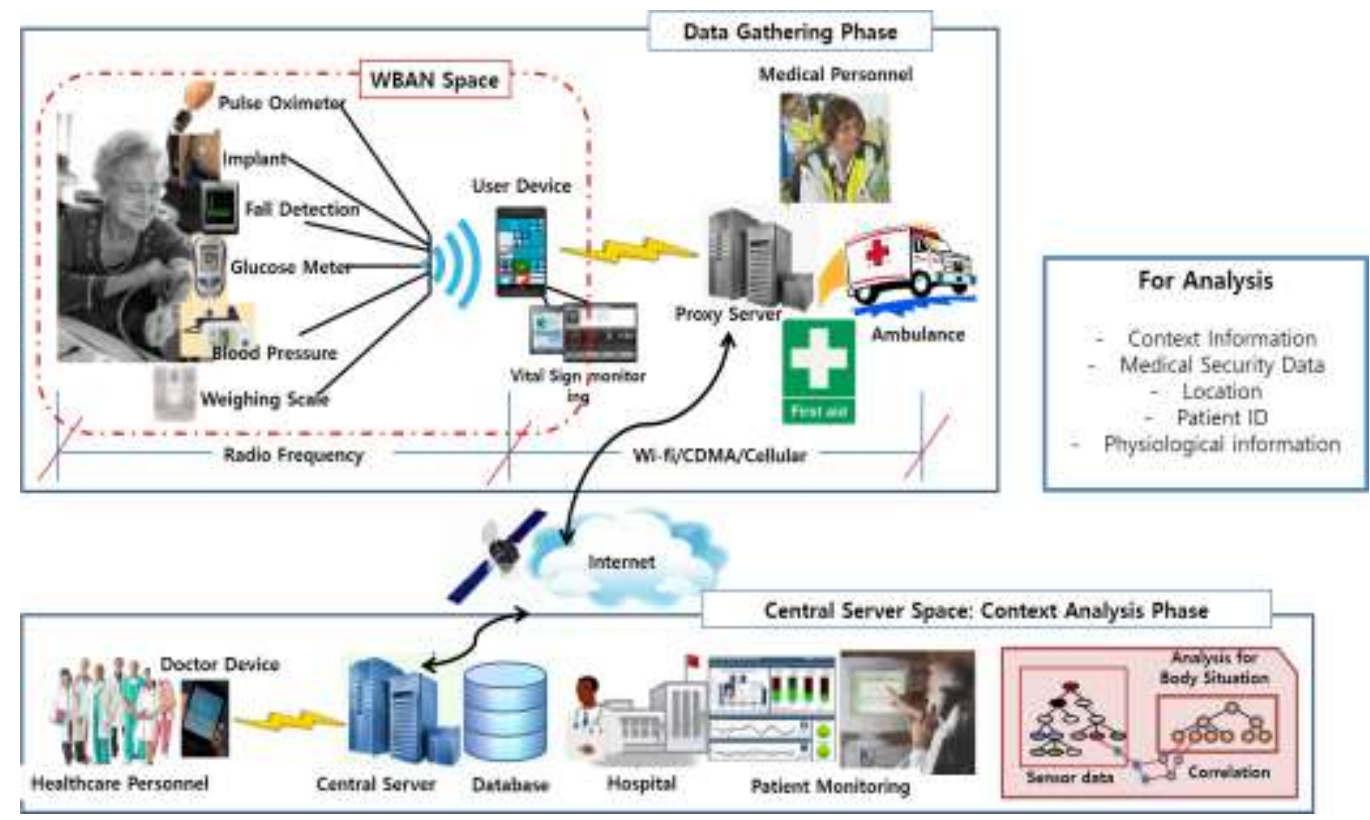

Figure 5. Data Gathering Phase

The user mobile device that aggregates the collected physiological information is also capable of checking the vital signs for health conditions that requires immediate treatment or diagnosis. The user mobile device is also capable of changing its point of attachment (PoA) across the different network environments. Its movement is supported by the optimization of FMIPv6 for a seamless connectivity and uninterrupted delivery of healthcare information as discussed in Section 3.

The collected physiological information will then be transmitted for context analysis in the local workstation located on healthcare centers or vehicles. The healthcare information includes the patient ID, its location, and the currently measured physiological information.

\subsection{Context Analysis Phase}

The context analysis phase is processed on the local workstation located in healthcare centers or vehicles. The received healthcare information will be matched onto the existing medical record history of the corresponding patient. The location and physiological information that was measured from the patient will be analyzed and reviewed for a certain change or changes of patient's health condition through context-aware processing and tag matching based on the patient's medical record history. The entire process for the context analysis is outlined in Figure 6.

In the case that the patient's current condition is stable, it simply updates the medical records of the patient in the Central Database Server and notifies the patient with his condition with a doctor's advice through his user mobile device. However, if the patient's current condition is not stable, that is, there are significant changes in the measured physiological information as compared to his medical history, it will then be determined if it is high risk or not. If classified as not a high risk, then a medical diagnosis and an emergent medication and treatment will be prescribed and provided to the patient. This is essentially important for emergency situations where an immediate response of medication and treatment is necessary. Conversely, if the patient's condition is determined to be a high risk, then a doctor will be chosen based on expertise (i.e., specialized doctor for heart, lungs, etc.), and the appropriate doctor will be giving the diagnosis, and long term medical prescription and treatment to the patient. The doctor will also give advice or instructions to the healthcare personnel or caregiver regarding the 
patient's current condition through their respective user mobile devices. The healthcare personnel update the medical records of the patient on the Central Database Server as soon as the instructions of the doctor/caregiver were completed.



Figure 6. Context Analysis Phase

For data security and privacy purposes, healthcare professionals (e.g., doctors, caregivers) can assign a security password for each patient's medical record. The IP packets for the healthcare information that are being transmitted is further encapsulated as part of the IP protocol to increase its security.

\section{Conclusion}

This paper has presented a context analysis of an IP-enabled u-Healthcare system. The IP-enabled u-Healthcare system utilizes the best features of wireless sensor networks (WSNs) to provide a remote real-time and continuous health monitoring for patients, specifically the elderly and those that suffering from high-risk or chronic diseases. The user mobile devices that is used for the aggregation of the measured physiological information for patients employs the mobility support based on the handover optimization of Fast Mobile Internet Protocol version 6 (FMIPv6). The user mobile devices can change its point of attachment across different wireless network environments with seamless connectivity and uninterrupted delivery of healthcare information. This guarantees the timely and accurate delivery of healthcare information and thus provides an efficient and effective provision of healthcare services as to the treatment, diagnosis, and prevention of high risk or chronic diseases. 


\section{Acknowledgments}

This paper has been supported by the 2017 Hannam University Research Fund.

\section{References}

[1] O. Chipara, C. Lu, T. C. Bailey, G.-C. Roman, "Reliable patient monitoring: A clinical study in a stepdown hospital unit", Dept. Comput. Sci. Eng., Washington Univ. St. Louis, St. Louis, MO, Tech. Rep. WUCSE-2009-82, (2009) December.

[2] G. Virone, A. Wood, L. Selavo, Q. Cao, L. Fang, T. Doan, Z. He, J. A. Stankovic, "An advanced wireless sensor network for health monitoring", in Proc. Transdisciplinary Conf. Distrib. Diagnosis Home Healthcare, (2006) April, pp. 95-100.

[3] D. Johnson, C. Perkins, J. Arkko, "Mobility Support in IPv6", Internet Engineering Task Force (IETF), RFC 3775, (2004) June.

[4] C. Perkins, D. Johnson, J. Arkko, "Mobility Support in IPv6", Internet Engineering Task Force (IETF), RFC 6275, ISSN: 2070-1721, (2011) July.

[5] R. Koodli, "Fast handovers for mobile IPv6", Internet Engineering Task Force (IETF), RFC 4068, (2005) July.

[6] V. Visoottiviseth, P. Ngamtura, "On the Performance of MIPv6 and FMIPv6 based on Real IPv6 applications over IEEE 802.11g Testbeds", Int. Symposium on Commun. and Inf. Technol. (ISCIT), (2010) October, pp. 1217-1222. 
International Journal of Control and Automation

Vol. 10, No. 11 (2017) 